\title{
Oscillatory behavior of second-order nonlinear neutral differential equations with distributed deviating arguments
}

Tongxing Li ${ }^{1 *}$, Blanka Baculíková ${ }^{2}$ and Jozef Džurina²

Dedicated to Professor Ivan Kiguradze

*Correspondence:

litongx2007@163.com

${ }^{1}$ Qingdao Technological University,

Feixian, Shandong 273400,

P.R. China

Full list of author information is

available at the end of the article

\begin{abstract}
We study oscillatory properties of a class of second-order nonlinear neutral functional differential equations with distributed deviating arguments. On the basis of less restrictive assumptions imposed on the neutral coefficient, some new criteria are presented. Three examples are provided to illustrate these results.

MSC: $34 \mathrm{C} 10 ; 34 \mathrm{~K} 11$
\end{abstract}

Keywords: oscillation; neutral differential equation; second-order equation; distributed deviating argument

\section{Introduction}

This paper is concerned with oscillation of the second-order nonlinear functional differential equation

$$
\left(r(t)\left|z^{\prime}(t)\right|^{\alpha-1} z^{\prime}(t)\right)^{\prime}+\int_{a}^{b} q(t, \xi)|x[g(t, \xi)]|^{\alpha-1} x[g(t, \xi)] \mathrm{d} \sigma(\xi)=0,
$$

where $t \geq t_{0}>0, \alpha \geq 1$ is a constant, and $z:=x+p \cdot x \circ \tau$. Throughout, we assume that the following hypotheses hold:

$\left(\mathrm{H}_{1}\right) \mathbb{I}:=\left[t_{0}, \infty\right), r, p \in \mathrm{C}^{1}(\mathbb{I}, \mathbb{R}), r(t)>0$, and $p(t) \geq 0$;

$\left(\mathrm{H}_{2}\right) q \in \mathrm{C}(\mathbb{I} \times[a, b],[0, \infty))$ and $q(t, \xi)$ is not eventually zero on any $\left[t_{\mu}, \infty\right) \times[a, b], t_{\mu} \in \mathbb{I}$;

$\left(\mathrm{H}_{3}\right) g \in \mathrm{C}(\mathbb{I} \times[a, b],[0, \infty))$, $\liminf _{t \rightarrow \infty} g(t, \xi)=\infty$, and $g(t, a) \leq g(t, \xi)$ for $\xi \in[a, b]$;

$\left(\mathrm{H}_{4}\right) \tau \in \mathrm{C}^{2}(\mathbb{I}, \mathbb{R}), \tau^{\prime}(t)>0, \lim _{t \rightarrow \infty} \tau(t)=\infty$, and $g(\tau(t), \xi)=\tau[g(t, \xi)]$;

$\left(\mathrm{H}_{5}\right) \sigma \in \mathrm{C}([a, b], \mathbb{R})$ is nondecreasing and the integral of (1.1) is taken in the sense of Riemann-Stieltijes.

By a solution of (1.1), we mean a function $x \in \mathrm{C}\left(\left[t_{x}, \infty\right), \mathbb{R}\right)$ for some $t_{x} \geq t_{0}$, which has the properties that $z \in C^{1}\left(\left[t_{x}, \infty\right), \mathbb{R}\right), r\left|z^{\prime}\right|^{\alpha-1} z^{\prime} \in C^{1}\left(\left[t_{x}, \infty\right), \mathbb{R}\right)$, and satisfies (1.1) on $\left[t_{x}, \infty\right)$. We restrict our attention to those solutions $x$ of $(1.1)$ which exist on $\left[t_{x}, \infty\right)$ and satisfy $\sup \{|x(t)|: t \geq T\}>0$ for any $T \geq t_{x}$. A solution $x$ of (1.1) is termed oscillatory if it is neither eventually positive nor eventually negative; otherwise, it is called nonoscillatory. Equation (1.1) is said to be oscillatory if all its solutions oscillate.

(2014 Li et al.; licensee Springer. This is an Open Access article distributed under the terms of the Creative Commons Attribution License (http://creativecommons.org/licenses/by/2.0), which permits unrestricted use, distribution, and reproduction in any medium, provided the original work is properly cited. 
As is well known, neutral differential equations have a great number of applications in electric networks. For instance, they are frequently used in the study of distributed networks containing lossless transmission lines, which rise in high speed computers, where the lossless transmission lines are used to interconnect switching circuits; see [1]. Hence, there has been much research activity concerning oscillatory and nonoscillatory behavior of solutions to different classes of neutral differential equations, we refer the reader to [2-30] and the references cited therein.

In the following, we present some background details that motivate our research. Recently, Baculíková and Lacková [6], Džurina and Hudáková [12], Li et al. [15, 18], and Sun et al. [22] established some oscillation criteria for the second-order half-linear neutral differential equation

$$
\left(r(t)\left|z^{\prime}(t)\right|^{\alpha-1} z^{\prime}(t)\right)^{\prime}+q(t)|x(\delta(t))|^{\alpha-1} x(\delta(t))=0,
$$

where $z:=x+p \cdot x \circ \tau$,

$$
0 \leq p(t)<1 \quad \text { or } \quad p(t)>1 .
$$

Baculíková and Džurina [4,5] and Li et al. [17] investigated oscillatory behavior of a second-order neutral differential equation

$$
\left(r(t)(x(t)+p(t) x[\tau(t)])^{\prime}\right)^{\prime}+q(t) x[\sigma(t)]=0,
$$

where

$$
0 \leq p(t) \leq p_{0}<\infty \quad \text { and } \quad \tau^{\prime}(t) \geq \tau_{0}>0 .
$$

Ye and $\mathrm{Xu}[26]$ and $\mathrm{Yu}$ and $\mathrm{Fu}$ [27] considered oscillation of the second-order differential equation

$$
(x(t)+p(t) x(t-\tau))^{\prime \prime}+\int_{a}^{b} q(t, \xi) x(g(t, \xi)) \mathrm{d} \sigma(\xi)=0 .
$$

Assuming $0 \leq p(t)<1$, Thandapani and Piramanantham [23], Wang [24], $\mathrm{Xu}$ and Weng [25], and Zhao and Meng [30] studied oscillation of an equation

$$
\left(r(t)(x(t)+p(t) x(t-\tau))^{\prime}\right)^{\prime}+\int_{a}^{b} q(t, \xi) f(x(g(t, \xi))) \mathrm{d} \sigma(\xi)=0 .
$$

As yet, there are few results regarding the study of oscillatory properties of (1.1) under the conditions $p(t) \geq 1$ or $\lim _{t \rightarrow \infty} p(t)=\infty$. Thereinto, Li and Thandapani [19] obtained several oscillation results for (1.1) in the case where (1.2) holds, $\sigma(\xi)=\xi$, and

$$
\int_{t_{0}}^{\infty} \frac{\mathrm{d} t}{r^{1 / \alpha}(t)}=\infty
$$

In the subsequent sections, we shall utilize the Riccati substitution technique and some inequalities to establish several new oscillation criteria for (1.1) assuming that (1.3) holds 
or

$$
\int_{t_{0}}^{\infty} \frac{\mathrm{d} t}{r^{1 / \alpha}(t)}<\infty
$$

All functional inequalities are assumed to hold eventually, that is, they are satisfied for all $t$ large enough.

\section{Main results}

In what follows, we use the following notation for the convenience of the reader:

$$
\begin{aligned}
& Q(t, \xi):=\min \{q(t, \xi), q(\tau(t), \xi)\}, \quad d_{+}(t):=\max \{0, d(t)\}, \\
& \phi(t):=\frac{\alpha p^{\prime}[h(t)] h^{\prime}(t)}{p[h(t)]}-\frac{\tau^{\prime \prime}(t)}{\tau^{\prime}(t)}, \quad \zeta(t):=\frac{\rho_{+}^{\prime}(t)}{\rho(t)}+\phi(t), \\
& \varphi(t):=\left(\frac{\rho_{+}^{\prime}(t)}{\rho(t)}\right)^{\alpha+1}+\frac{p^{\alpha}[h(t)]\left(\zeta_{+}(t)\right)^{\alpha+1}}{\tau^{\prime}(t)}, \quad \text { and } \quad \delta(t):=\int_{\eta(t)}^{\infty} \frac{\mathrm{d} s}{r^{1 / \alpha}(s)},
\end{aligned}
$$

where $h, \rho$, and $\eta$ will be specified later.

Theorem 2.1 Assume $\left(\mathrm{H}_{1}\right)-\left(\mathrm{H}_{5}\right),(1.3)$, and let $g(t, a) \in \mathrm{C}^{1}(\mathbb{I}, \mathbb{R}), g^{\prime}(t, a)>0, g(t, a) \leq t$, and $g(t, a) \leq \tau(t)$ for $t \in \mathbb{I}$. Suppose further that there exists a real-valued function $h \in C^{1}(\mathbb{I}, \mathbb{R})$ such that $p[g(t, \xi)] \leq p[h(t)]$ for $t \in \mathbb{I}$ and $\xi \in[a, b]$. If there exists a real-valued function $\rho \in \mathrm{C}^{1}(\mathbb{I},(0, \infty))$ such that

$$
\limsup _{t \rightarrow \infty} \int_{t_{0}}^{t} \rho(s)\left[\frac{\int_{a}^{b} Q(s, \xi) \mathrm{d} \sigma(\xi)}{2^{\alpha-1}}-\frac{r[g(s, a)] \varphi(s)}{(\alpha+1)^{\alpha+1}\left(g^{\prime}(s, a)\right)^{\alpha}}\right] \mathrm{d} s=\infty
$$

then (1.1) is oscillatory.

Proof Let $x$ be a nonoscillatory solution of (1.1). Without loss of generality, we assume that there exists a $t_{1} \in \mathbb{I}$ such that $x(t)>0, x[\tau(t)]>0$, and $x[g(t, \xi)]>0$ for all $t \geq t_{1}$ and $\xi \in[a, b]$. Then $z(t)>0$. Applying (1.1), one has, for all sufficiently large $t$,

$$
\begin{gathered}
\left(r(t)\left|z^{\prime}(t)\right|^{\alpha-1} z^{\prime}(t)\right)^{\prime}+\int_{a}^{b} q(t, \xi) x^{\alpha}[g(t, \xi)] \mathrm{d} \sigma(\xi) \\
+\int_{a}^{b} q(\tau(t), \xi) p^{\alpha}[h(t)] x^{\alpha}[g(\tau(t), \xi)] \mathrm{d} \sigma(\xi) \\
+\frac{p^{\alpha}[h(t)]}{\tau^{\prime}(t)}\left(r[\tau(t)]\left|z^{\prime}[\tau(t)]\right|^{\alpha-1} z^{\prime}[\tau(t)]\right)^{\prime}=0 .
\end{gathered}
$$

Using the inequality (see [5, Lemma 1$]$ )

$$
(A+B)^{\alpha} \leq 2^{\alpha-1}\left(A^{\alpha}+B^{\alpha}\right), \quad \text { for } A \geq 0, B \geq 0, \text { and } \alpha \geq 1,
$$


the definition of $z, g(\tau(t), \xi)=\tau[g(t, \xi)]$, and $p[g(t, \xi)] \leq p[h(t)]$, we conclude that

$$
\begin{gathered}
\left(r(t)\left|z^{\prime}(t)\right|^{\alpha-1} z^{\prime}(t)\right)^{\prime}+\frac{1}{2^{\alpha-1}} \int_{a}^{b} Q(t, \xi) z^{\alpha}[g(t, \xi)] \mathrm{d} \sigma(\xi) \\
+\frac{p^{\alpha}[h(t)]}{\tau^{\prime}(t)}\left(r[\tau(t)]\left|z^{\prime}[\tau(t)]\right|^{\alpha-1} z^{\prime}[\tau(t)]\right)^{\prime} \leq 0 .
\end{gathered}
$$

By virtue of (1.1), we get

$$
\left(r(t)\left|z^{\prime}(t)\right|^{\alpha-1} z^{\prime}(t)\right)^{\prime} \leq 0, \quad t \geq t_{1}
$$

Thus, $r\left|z^{\prime}\right|^{\alpha-1} z^{\prime}$ is nonincreasing. Now we have two possible cases for the sign of $z^{\prime}$ : (i) $z^{\prime}<0$ eventually, or (ii) $z^{\prime}>0$ eventually.

(i) Assume that $z^{\prime}(t)<0$ for $t \geq t_{2} \geq t_{1}$. Then we have by (2.3)

$$
r(t)\left|z^{\prime}(t)\right|^{\alpha-1} z^{\prime}(t) \leq r\left(t_{2}\right)\left|z^{\prime}\left(t_{2}\right)\right|^{\alpha-1} z^{\prime}\left(t_{2}\right)<0, \quad t \geq t_{2},
$$

which yields

$$
z(t) \leq z\left(t_{2}\right)-r^{1 / \alpha}\left(t_{2}\right)\left|z^{\prime}\left(t_{2}\right)\right| \int_{t_{2}}^{t} r^{-1 / \alpha}(s) \mathrm{d} s
$$

Then we obtain $\lim _{t \rightarrow \infty} z(t)=-\infty$ due to (1.3), which is a contradiction.

(ii) Assume that $z^{\prime}(t)>0$ for $t \geq t_{2} \geq t_{1}$. It follows from (2.2) and $g(t, \xi) \geq g(t, a)$ that

$$
\begin{gathered}
\left(r(t)\left(z^{\prime}(t)\right)^{\alpha}\right)^{\prime}+\frac{p^{\alpha}[h(t)]}{\tau^{\prime}(t)}\left(r[\tau(t)]\left(z^{\prime}[\tau(t)]\right)^{\alpha}\right)^{\prime} \\
+\frac{1}{2^{\alpha-1}} z^{\alpha}[g(t, a)] \int_{a}^{b} Q(t, \xi) \mathrm{d} \sigma(\xi) \leq 0 .
\end{gathered}
$$

We define a Riccati substitution

$$
\omega(t):=\rho(t) \frac{r(t)\left(z^{\prime}(t)\right)^{\alpha}}{(z[g(t, a)])^{\alpha}}, \quad t \geq t_{2} .
$$

Then $\omega(t)>0$. From $(2.3)$ and $g(t, a) \leq t$, we have

$$
z^{\prime}[g(t, a)] \geq(r(t) / r[g(t, a)])^{1 / \alpha} z^{\prime}(t)
$$

Differentiating (2.5), we get

$$
\begin{aligned}
\omega^{\prime}(t)= & \rho^{\prime}(t) \frac{r(t)\left(z^{\prime}(t)\right)^{\alpha}}{(z[g(t, a)])^{\alpha}}+\rho(t) \frac{\left(r(t)\left(z^{\prime}(t)\right)^{\alpha}\right)^{\prime}}{(z[g(t, a)])^{\alpha}} \\
& -\alpha \rho(t) \frac{r(t)\left(z^{\prime}(t)\right)^{\alpha} z^{\alpha-1}[g(t, a)] z^{\prime}[g(t, a)] g^{\prime}(t, a)}{(z[g(t, a)])^{2 \alpha}} .
\end{aligned}
$$

Therefore, by (2.5), (2.6), and (2.7), we see that

$$
\omega^{\prime}(t) \leq \frac{\rho^{\prime}(t)}{\rho(t)} \omega(t)+\rho(t) \frac{\left(r(t)\left(z^{\prime}(t)\right)^{\alpha}\right)^{\prime}}{(z[g(t, a)])^{\alpha}}-\frac{\alpha g^{\prime}(t, a)}{\rho^{1 / \alpha}(t) r^{1 / \alpha}[g(t, a)]} \omega^{(\alpha+1) / \alpha}(t) .
$$


Similarly, we introduce another Riccati transformation:

$$
v(t):=\rho(t) \frac{r[\tau(t)]\left(z^{\prime}[\tau(t)]\right)^{\alpha}}{(z[g(t, a)])^{\alpha}}, \quad t \geq t_{2} .
$$

Then $v(t)>0$. From (2.3) and $g(t, a) \leq \tau(t)$, we obtain

$$
z^{\prime}[g(t, a)] \geq(r[\tau(t)] / r[g(t, a)])^{1 / \alpha} z^{\prime}[\tau(t)]
$$

Differentiating (2.9), we have

$$
\begin{aligned}
v^{\prime}(t)= & \rho^{\prime}(t) \frac{r[\tau(t)]\left(z^{\prime}[\tau(t)]\right)^{\alpha}}{(z[g(t, a)])^{\alpha}}+\rho(t) \frac{\left(r[\tau(t)]\left(z^{\prime}[\tau(t)]\right)^{\alpha}\right)^{\prime}}{(z[g(t, a)])^{\alpha}} \\
& -\alpha \rho(t) \frac{r[\tau(t)]\left(z^{\prime}[\tau(t)]\right)^{\alpha} z^{\alpha-1}[g(t, a)] z^{\prime}[g(t, a)] g^{\prime}(t, a)}{(z[g(t, a)])^{2 \alpha}} .
\end{aligned}
$$

Therefore, by (2.9), (2.10), and (2.11), we find

$$
v^{\prime}(t) \leq \frac{\rho^{\prime}(t)}{\rho(t)} v(t)+\rho(t) \frac{\left(r[\tau(t)]\left(z^{\prime}[\tau(t)]\right)^{\alpha}\right)^{\prime}}{(z[g(t, a)])^{\alpha}}-\frac{\alpha g^{\prime}(t, a)}{\rho^{1 / \alpha}(t) r^{1 / \alpha}[g(t, a)]} v^{(\alpha+1) / \alpha}(t) .
$$

Combining (2.8) and (2.12), we get

$$
\begin{aligned}
\omega^{\prime}(t) & +\frac{p^{\alpha}[h(t)]}{\tau^{\prime}(t)} v^{\prime}(t) \\
\leq & \rho(t) \frac{\left(r(t)\left(z^{\prime}(t)\right)^{\alpha}\right)^{\prime}+\frac{p^{\alpha}[h(t)]}{\tau^{\prime}(t)}\left(r[\tau(t)]\left(z^{\prime}[\tau(t)]\right)^{\alpha}\right)^{\prime}}{(z[g(t, a)])^{\alpha}}+\frac{\rho^{\prime}(t)}{\rho(t)} \omega(t) \\
& -\frac{\alpha g^{\prime}(t, a)}{\rho^{1 / \alpha}(t) r^{1 / \alpha}[g(t, a)]} \omega^{(\alpha+1) / \alpha}(t)+\frac{p^{\alpha}[h(t)]}{\tau^{\prime}(t)} \frac{\rho^{\prime}(t)}{\rho(t)} v(t) \\
& -\frac{p^{\alpha}[h(t)]}{\tau^{\prime}(t)} \frac{\alpha g^{\prime}(t, a)}{\rho^{1 / \alpha}(t) r^{1 / \alpha}[g(t, a)]} v^{(\alpha+1) / \alpha}(t) .
\end{aligned}
$$

It follows from (2.4) that

$$
\begin{aligned}
\omega^{\prime}(t)+\frac{p^{\alpha}[h(t)]}{\tau^{\prime}(t)} v^{\prime}(t) \leq & -\frac{\rho(t)}{2^{\alpha-1}} \int_{a}^{b} Q(t, \xi) \mathrm{d} \sigma(\xi)+\frac{\rho_{+}^{\prime}(t)}{\rho(t)} \omega(t) \\
& -\frac{\alpha g^{\prime}(t, a)}{\rho^{1 / \alpha}(t) r^{1 / \alpha}[g(t, a)]} \omega^{(\alpha+1) / \alpha}(t)+\frac{p^{\alpha}[h(t)]}{\tau^{\prime}(t)} \frac{\rho_{+}^{\prime}(t)}{\rho(t)} v(t) \\
& -\frac{p^{\alpha}[h(t)]}{\tau^{\prime}(t)} \frac{\alpha g^{\prime}(t, a)}{\rho^{1 / \alpha}(t) r^{1 / \alpha}[g(t, a)]} v^{(\alpha+1) / \alpha}(t) .
\end{aligned}
$$

Integrating the latter inequality from $t_{2}$ to $t$, we obtain

$$
\begin{gathered}
\omega(t)-\omega\left(t_{2}\right)+\frac{p^{\alpha}[h(t)]}{\tau^{\prime}(t)} v(t)-\frac{p^{\alpha}\left[h\left(t_{2}\right)\right]}{\tau^{\prime}\left(t_{2}\right)} v\left(t_{2}\right) \\
\leq-\int_{t_{2}}^{t} \frac{\rho(s)}{2^{\alpha-1}} \int_{a}^{b} Q(s, \xi) \mathrm{d} \sigma(\xi) \mathrm{d} s
\end{gathered}
$$




$$
\begin{aligned}
& +\int_{t_{2}}^{t}\left[\frac{\rho_{+}^{\prime}(s)}{\rho(s)} \omega(s)-\frac{\alpha g^{\prime}(s, a)}{\rho^{1 / \alpha}(s) r^{1 / \alpha}[g(s, a)]} \omega^{(\alpha+1) / \alpha}(s)\right] \mathrm{d} s \\
& +\int_{t_{2}}^{t} \frac{p^{\alpha}[h(s)]}{\tau^{\prime}(s)}\left\{\left[\frac{\rho_{+}^{\prime}(s)}{\rho(s)}+\phi(s)\right]_{+} v(s)-\frac{\alpha g^{\prime}(s, a)}{\rho^{1 / \alpha}(s) r^{1 / \alpha}[g(s, a)]} v^{(\alpha+1) / \alpha}(s)\right\} \mathrm{d} s .
\end{aligned}
$$

Define

$$
\begin{aligned}
& A:=\left[\frac{\alpha g^{\prime}(t, a)}{\rho^{1 / \alpha}(t) r^{1 / \alpha}[g(t, a)]}\right]^{\alpha /(\alpha+1)} \omega(t) \quad \text { and } \\
& B:=\left[\frac{\alpha}{\alpha+1} \frac{\rho_{+}^{\prime}(t)}{\rho(t)}\left[\frac{\alpha g^{\prime}(t, a)}{\rho^{1 / \alpha}(t) r^{1 / \alpha}[g(t, a)]}\right]^{-\alpha /(\alpha+1)}\right]^{\alpha} .
\end{aligned}
$$

Using the inequality

$$
\frac{\alpha+1}{\alpha} A B^{1 / \alpha}-A^{(\alpha+1) / \alpha} \leq \frac{1}{\alpha} B^{(\alpha+1) / \alpha}, \quad \text { for } A \geq 0 \text { and } B \geq 0,
$$

we get

$$
\frac{\rho_{+}^{\prime}(t)}{\rho(t)} \omega(t)-\frac{\alpha g^{\prime}(t, a)}{\rho^{1 / \alpha}(t) r^{1 / \alpha}[g(t, a)]} \omega^{(\alpha+1) / \alpha}(t) \leq \frac{1}{(\alpha+1)^{\alpha+1}} \frac{r[g(t, a)]\left(\rho_{+}^{\prime}(t)\right)^{\alpha+1}}{\left(\rho(t) g^{\prime}(t, a)\right)^{\alpha}} .
$$

On the other hand, define

$$
\begin{aligned}
& A:=\left[\frac{\alpha g^{\prime}(t, a)}{\rho^{1 / \alpha}(t) r^{1 / \alpha}[g(t, a)]}\right]^{\alpha /(\alpha+1)} v(t) \text { and } \\
& B:=\left[\frac{\alpha}{\alpha+1} \zeta_{+}(t)\left[\frac{\alpha g^{\prime}(t, a)}{\rho^{1 / \alpha}(t) r^{1 / \alpha}[g(t, a)]}\right]^{-\alpha /(\alpha+1)}\right]^{\alpha} .
\end{aligned}
$$

Then we have by (2.14)

$$
\zeta_{+}(t) v(t)-\frac{\alpha g^{\prime}(t, a)}{\rho^{1 / \alpha}(t) r^{1 / \alpha}[g(t, a)]} v^{(\alpha+1) / \alpha}(t) \leq \frac{1}{(\alpha+1)^{\alpha+1}} \frac{r[g(t, a)]\left(\zeta_{+}(t)\right)^{\alpha+1} \rho(t)}{\left(g^{\prime}(t, a)\right)^{\alpha}} .
$$

Thus, from (2.13), we get

$$
\begin{aligned}
\omega(t) & -\omega\left(t_{2}\right)+\frac{p^{\alpha}[h(t)]}{\tau^{\prime}(t)} v(t)-\frac{p^{\alpha}\left[h\left(t_{2}\right)\right]}{\tau^{\prime}\left(t_{2}\right)} v\left(t_{2}\right) \\
\leq & -\int_{t_{2}}^{t} \rho(s)\left\{\frac{\int_{a}^{b} Q(s, \xi) \mathrm{d} \sigma(\xi)}{2^{\alpha-1}}-\frac{r[g(s, a)]}{(\alpha+1)^{\alpha+1}\left(g^{\prime}(s, a)\right)^{\alpha}}\right. \\
& \left.\times\left[\left(\frac{\rho_{+}^{\prime}(s)}{\rho(s)}\right)^{\alpha+1}+\frac{p^{\alpha}[h(s)]\left(\zeta_{+}(s)\right)^{\alpha+1}}{\tau^{\prime}(s)}\right]\right\} \mathrm{d} s,
\end{aligned}
$$

which contradicts (2.1). This completes the proof.

Assuming (1.2), where $p_{0}$ and $\tau_{0}$ are constants, we obtain the following result.

Theorem 2.2 Suppose $\left(\mathrm{H}_{1}\right)-\left(\mathrm{H}_{5}\right),(1.2),(1.3)$, and let $g(t, a) \in \mathrm{C}^{1}(\mathbb{I}, \mathbb{R}), g^{\prime}(t, a)>0, g(t, a) \leq$ $t$, and $g(t, a) \leq \tau(t)$ for $t \in \mathbb{I}$. If there exists a real-valued function $\rho \in C^{1}(\mathbb{I},(0, \infty))$ such 
that

$$
\limsup _{t \rightarrow \infty} \int_{t_{0}}^{t}\left[\frac{\rho(s) \int_{a}^{b} Q(s, \xi) \mathrm{d} \sigma(\xi)}{2^{\alpha-1}}-\frac{1+\frac{p_{0}^{\alpha}}{\tau_{0}}}{(\alpha+1)^{\alpha+1}} \frac{r[g(s, a)]\left(\rho_{+}^{\prime}(s)\right)^{\alpha+1}}{\left(\rho(s) g^{\prime}(s, a)\right)^{\alpha}}\right] \mathrm{d} s=\infty,
$$

then (1.1) is oscillatory.

Proof As above, let $x$ be an eventually positive solution of (1.1). Proceeding as in the proof of Theorem 2.1, we have $z^{\prime}(t)>0,(2.3)$, and (2.4) for all sufficiently large $t$. Using (1.2), (2.3), and (2.4), we obtain

$$
\begin{gathered}
\left(r(t)\left(z^{\prime}(t)\right)^{\alpha}\right)^{\prime}+\frac{p_{0}^{\alpha}}{\tau_{0}}\left(r[\tau(t)]\left(z^{\prime}[\tau(t)]\right)^{\alpha}\right)^{\prime} \\
\quad+\frac{1}{2^{\alpha-1}} z^{\alpha}[g(t, a)] \int_{a}^{b} Q(s, \xi) \mathrm{d} \sigma(\xi) \leq 0 .
\end{gathered}
$$

The remainder of the proof is similar to that of Theorem 2.1, and hence it is omitted.

Theorem 2.3 Suppose we have $\left(\mathrm{H}_{1}\right)-\left(\mathrm{H}_{5}\right),(1.3)$, and let $\tau(t) \leq t$ and $g(t, a) \geq \tau(t)$ for $t \in \mathbb{I}$. Assume also that there exists a real-valued function $h \in C^{1}(\mathbb{I}, \mathbb{R})$ such that $p[g(t, \xi)] \leq$ $p[h(t)]$ for $t \in \mathbb{I}$ and $\xi \in[a, b]$. If there exists a real-valued function $\rho \in \mathrm{C}^{1}(\mathbb{I},(0, \infty))$ such that

$$
\limsup _{t \rightarrow \infty} \int_{t_{0}}^{t} \rho(s)\left[\frac{\int_{a}^{b} Q(s, \xi) \mathrm{d} \sigma(\xi)}{2^{\alpha-1}}-\frac{r[\tau(s)] \varphi(s)}{(\alpha+1)^{\alpha+1}\left(\tau^{\prime}(s)\right)^{\alpha}}\right] \mathrm{d} s=\infty
$$

then (1.1) is oscillatory.

Proof Let $x$ be a nonoscillatory solution of (1.1). Without loss of generality, we assume that there exists a $t_{1} \in \mathbb{I}$ such that $x(t)>0, x[\tau(t)]>0$, and $x[g(t, \xi)]>0$ for all $t \geq t_{1}$ and $\xi \in$ $[a, b]$. As in the proof of Theorem 2.1, we obtain (2.3) and (2.4). In view of (2.3), $r\left|z^{\prime}\right|^{\alpha-1} z^{\prime}$ is nonincreasing. Now we have two possible cases for the sign of $z^{\prime}$ : (i) $z^{\prime}<0$ eventually, or (ii) $z^{\prime}>0$ eventually.

(i) Suppose that $z^{\prime}(t)<0$ for $t \geq t_{2} \geq t_{1}$. Then, with a proof similar to the proof of case (i) in Theorem 2.1, we obtain a contradiction.

(ii) Suppose that $z^{\prime}(t)>0$ for $t \geq t_{2} \geq t_{1}$. We define a Riccati substitution

$$
\omega(t):=\rho(t) \frac{r(t)\left(z^{\prime}(t)\right)^{\alpha}}{(z[\tau(t)])^{\alpha}}, \quad t \geq t_{2} .
$$

Then $\omega(t)>0$. From $(2.3)$ and $\tau(t) \leq t$, we have

$$
z^{\prime}[\tau(t)] \geq(r(t) / r[\tau(t)])^{1 / \alpha} z^{\prime}(t) .
$$

Differentiating (2.18), we obtain

$$
\begin{aligned}
\omega^{\prime}(t)= & \rho^{\prime}(t) \frac{r(t)\left(z^{\prime}(t)\right)^{\alpha}}{(z[\tau(t)])^{\alpha}}+\rho(t) \frac{\left(r(t)\left(z^{\prime}(t)\right)^{\alpha}\right)^{\prime}}{(z[\tau(t)])^{\alpha}} \\
& -\alpha \rho(t) \frac{r(t)\left(z^{\prime}(t)\right)^{\alpha} z^{\alpha-1}[\tau(t)] z^{\prime}[\tau(t)] \tau^{\prime}(t)}{(z[\tau(t)])^{2 \alpha}} .
\end{aligned}
$$


Therefore, by (2.18), (2.19), and (2.20), we see that

$$
\omega^{\prime}(t) \leq \frac{\rho^{\prime}(t)}{\rho(t)} \omega(t)+\rho(t) \frac{\left(r(t)\left(z^{\prime}(t)\right)^{\alpha}\right)^{\prime}}{(z[\tau(t)])^{\alpha}}-\frac{\alpha \tau^{\prime}(t)}{\rho^{1 / \alpha}(t) r^{1 / \alpha}[\tau(t)]} \omega^{(\alpha+1) / \alpha}(t) .
$$

Similarly, we introduce another Riccati substitution:

$$
v(t):=\rho(t) \frac{r[\tau(t)]\left(z^{\prime}[\tau(t)]\right)^{\alpha}}{(z[\tau(t)])^{\alpha}}, \quad t \geq t_{2} .
$$

Then $v(t)>0$. Differentiating (2.22), we have

$$
\begin{aligned}
v^{\prime}(t)= & \rho^{\prime}(t) \frac{r[\tau(t)]\left(z^{\prime}[\tau(t)]\right)^{\alpha}}{(z[\tau(t)])^{\alpha}}+\rho(t) \frac{\left(r[\tau(t)]\left(z^{\prime}[\tau(t)]\right)^{\alpha}\right)^{\prime}}{(z[\tau(t)])^{\alpha}} \\
& -\alpha \rho(t) \frac{r[\tau(t)]\left(z^{\prime}[\tau(t)]\right)^{\alpha} z^{\alpha-1}[\tau(t)] z^{\prime}[\tau(t)] \tau^{\prime}(t)}{(z[\tau(t)])^{2 \alpha}} .
\end{aligned}
$$

Therefore, by (2.22) and (2.23), we get

$$
v^{\prime}(t)=\frac{\rho^{\prime}(t)}{\rho(t)} v(t)+\rho(t) \frac{\left(r[\tau(t)]\left(z^{\prime}[\tau(t)]\right)^{\alpha}\right)^{\prime}}{(z[\tau(t)])^{\alpha}}-\frac{\alpha \tau^{\prime}(t)}{\rho^{1 / \alpha}(t) r^{1 / \alpha}[\tau(t)]} v^{(\alpha+1) / \alpha}(t) .
$$

Combining (2.21) and (2.24), we have

$$
\begin{aligned}
\omega^{\prime}(t)+\frac{p^{\alpha}[h(t)]}{\tau^{\prime}(t)} v^{\prime}(t) \leq & \rho(t) \frac{\left(r(t)\left(z^{\prime}(t)\right)^{\alpha}\right)^{\prime}+\frac{p^{\alpha}[h(t)]}{\tau^{\prime}(t)}\left(r[\tau(t)]\left(z^{\prime}[\tau(t)]\right)^{\alpha}\right)^{\prime}}{(z[\tau(t)])^{\alpha}}+\frac{\rho^{\prime}(t)}{\rho(t)} \omega(t) \\
& -\frac{\alpha \tau^{\prime}(t)}{\rho^{1 / \alpha}(t) r^{1 / \alpha}[\tau(t)]} \omega^{(\alpha+1) / \alpha}(t)+\frac{p^{\alpha}[h(t)]}{\tau^{\prime}(t)} \frac{\rho^{\prime}(t)}{\rho(t)} v(t) \\
& -\frac{p^{\alpha}[h(t)]}{\tau^{\prime}(t)} \frac{\alpha \tau^{\prime}(t)}{\rho^{1 / \alpha}(t) r^{1 / \alpha}[\tau(t)]} v^{(\alpha+1) / \alpha}(t) .
\end{aligned}
$$

It follows from (2.4) and $g(t, a) \geq \tau(t)$ that

$$
\begin{aligned}
\omega^{\prime}(t)+\frac{p^{\alpha}[h(t)]}{\tau^{\prime}(t)} v^{\prime}(t) \leq & -\frac{\rho(t)}{2^{\alpha-1}} \int_{a}^{b} Q(t, \xi) \mathrm{d} \sigma(\xi)+\frac{\rho_{+}^{\prime}(t)}{\rho(t)} \omega(t) \\
& -\frac{\alpha \tau^{\prime}(t)}{\rho^{1 / \alpha}(t) r^{1 / \alpha}[\tau(t)]} \omega^{(\alpha+1) / \alpha}(t)+\frac{p^{\alpha}[h(t)]}{\tau^{\prime}(t)} \frac{\rho_{+}^{\prime}(t)}{\rho(t)} v(t) \\
& -\frac{p^{\alpha}[h(t)]}{\tau^{\prime}(t)} \frac{\alpha \tau^{\prime}(t)}{\rho^{1 / \alpha}(t) r^{1 / \alpha}[\tau(t)]} v^{(\alpha+1) / \alpha}(t) .
\end{aligned}
$$

Integrating the latter inequality from $t_{2}$ to $t$, we obtain

$$
\begin{aligned}
\omega(t) & -\omega\left(t_{2}\right)+\frac{p^{\alpha}[h(t)]}{\tau^{\prime}(t)} v(t)-\frac{p^{\alpha}\left[h\left(t_{2}\right)\right]}{\tau^{\prime}\left(t_{2}\right)} v\left(t_{2}\right) \\
\leq & -\int_{t_{2}}^{t} \frac{\rho(s)}{2^{\alpha-1}} \int_{a}^{b} Q(s, \xi) \mathrm{d} \sigma(\xi) \mathrm{d} s+\int_{t_{2}}^{t}\left[\frac{\rho_{+}^{\prime}(s)}{\rho(s)} \omega(s)-\frac{\alpha \tau^{\prime}(s)}{\rho^{1 / \alpha}(s) r^{1 / \alpha}[\tau(s)]} \omega^{(\alpha+1) / \alpha}(s)\right] \mathrm{d} s \\
& +\int_{t_{2}}^{t} \frac{p^{\alpha}[h(s)]}{\tau^{\prime}(s)}\left\{\left[\frac{\rho_{+}^{\prime}(s)}{\rho(s)}+\phi(s)\right]_{+} v(s)-\frac{\alpha \tau^{\prime}(s)}{\rho^{1 / \alpha}(s) r^{1 / \alpha}[\tau(s)]} v^{(\alpha+1) / \alpha}(s)\right\} \mathrm{d} s .
\end{aligned}
$$


Define

$$
\begin{aligned}
& A:=\left[\frac{\alpha \tau^{\prime}(t)}{\rho^{1 / \alpha}(t) r^{1 / \alpha}[\tau(t)]}\right]^{\alpha /(\alpha+1)} \omega(t) \quad \text { and } \\
& B:=\left[\frac{\alpha}{\alpha+1} \frac{\rho_{+}^{\prime}(t)}{\rho(t)}\left[\frac{\alpha \tau^{\prime}(t)}{\rho^{1 / \alpha}(t) r^{1 / \alpha}[\tau(t)]}\right]^{-\alpha /(\alpha+1)}\right]^{\alpha} .
\end{aligned}
$$

Using inequality (2.14), we have

$$
\frac{\rho_{+}^{\prime}(t)}{\rho(t)} \omega(t)-\frac{\alpha \tau^{\prime}(t)}{\rho^{1 / \alpha}(t) r^{1 / \alpha}[\tau(t)]} \omega^{(\alpha+1) / \alpha}(t) \leq \frac{1}{(\alpha+1)^{\alpha+1}} \frac{r[\tau(t)]\left(\rho_{+}^{\prime}(t)\right)^{\alpha+1}}{\left(\rho(t) \tau^{\prime}(t)\right)^{\alpha}} .
$$

On the other hand, define

$$
\begin{aligned}
& A:=\left[\frac{\alpha \tau^{\prime}(t)}{\rho^{1 / \alpha}(t) r^{1 / \alpha}[\tau(t)]}\right]^{\alpha /(\alpha+1)} v(t) \text { and } \\
& B:=\left[\frac{\alpha}{\alpha+1} \zeta_{+}(t)\left[\frac{\alpha \tau^{\prime}(t)}{\rho^{1 / \alpha}(t) r^{1 / \alpha}[\tau(t)]}\right]^{-\alpha /(\alpha+1)}\right]^{\alpha} .
\end{aligned}
$$

Then, by (2.14), we obtain

$$
\zeta_{+}(t) v(t)-\frac{\alpha \tau^{\prime}(t)}{\rho^{1 / \alpha}(t) r^{1 / \alpha}[\tau(t)]} v^{(\alpha+1) / \alpha}(t) \leq \frac{1}{(\alpha+1)^{\alpha+1}} \frac{r[\tau(t)]\left(\zeta_{+}(t)\right)^{\alpha+1} \rho(t)}{\left(\tau^{\prime}(t)\right)^{\alpha}} .
$$

Thus, from (2.25), we get

$$
\begin{aligned}
\omega(t) & -\omega\left(t_{2}\right)+\frac{p^{\alpha}[h(t)]}{\tau^{\prime}(t)} v(t)-\frac{p^{\alpha}\left[h\left(t_{2}\right)\right]}{\tau^{\prime}\left(t_{2}\right)} v\left(t_{2}\right) \\
\leq & -\int_{t_{2}}^{t} \rho(s)\left\{\frac{\int_{a}^{b} Q(s, \xi) \mathrm{d} \sigma(\xi)}{2^{\alpha-1}}-\frac{r[\tau(s)]}{(\alpha+1)^{\alpha+1}\left(\tau^{\prime}(s)\right)^{\alpha}}\right. \\
& \left.\times\left[\left(\frac{\rho_{+}^{\prime}(s)}{\rho(s)}\right)^{\alpha+1}+\frac{p^{\alpha}[h(s)]\left(\zeta_{+}(s)\right)^{\alpha+1}}{\tau^{\prime}(s)}\right]\right\} \mathrm{d} s,
\end{aligned}
$$

which contradicts (2.17). This completes the proof.

Assuming we have (1.2), where $p_{0}$ and $\tau_{0}$ are constants, we get the following result.

Theorem 2.4 Suppose we have $\left(\mathrm{H}_{1}\right)-\left(\mathrm{H}_{5}\right),(1.2),(1.3)$, and let $\tau(t) \leq t$ and $g(t, a) \geq \tau(t)$ for $t \in \mathbb{I}$. If there exists a real-valued function $\rho \in \mathrm{C}^{1}(\mathbb{I},(0, \infty))$ such that

$$
\limsup _{t \rightarrow \infty} \int_{t_{0}}^{t}\left[\frac{\rho(s) \int_{a}^{b} Q(s, \xi) \mathrm{d} \sigma(\xi)}{2^{\alpha-1}}-\frac{1}{(\alpha+1)^{\alpha+1}}\left(1+\frac{p_{0}^{\alpha}}{\tau_{0}}\right) \frac{r[\tau(s)]\left(\rho_{+}^{\prime}(s)\right)^{\alpha+1}}{\left(\tau_{0} \rho(s)\right)^{\alpha}}\right] \mathrm{d} s=\infty,
$$

then (1.1) is oscillatory.

Proof Assume again that $x$ is an eventually positive solution of (1.1). As in the proof of Theorem 2.1, we have $z^{\prime}(t)>0,(2.3)$, and (2.4) for all sufficiently large $t$. By virtue of (1.2), 
(2.3), and (2.4), we have (2.16) for all sufficiently large $t$. The rest of the proof is similar to that of Theorem 2.3, and so it is omitted.

In the following, we present some oscillation criteria for (1.1) in the case where (1.4) holds.

Theorem 2.5 Suppose we have $\left(\mathrm{H}_{1}\right)-\left(\mathrm{H}_{5}\right),(1.2),(1.4)$, and let $g(t, a) \in \mathrm{C}^{1}(\mathbb{I}, \mathbb{R}), g^{\prime}(t, a)>0$, $g(t, a) \leq \tau(t) \leq t$ for $t \in \mathbb{I}$, and $g(t, \xi) \leq g(t, b)$ for $\xi \in[a, b]$. Assume further that there exists a real-valued function $\rho \in C^{1}(\mathbb{I},(0, \infty))$ such that $(2.15)$ is satisfied. If there exists a realvalued function $\eta \in C^{1}(\mathbb{I}, \mathbb{R})$ such that $\eta(t) \geq t, \eta(t) \geq g(t, b), \eta^{\prime}(t)>0$ for $t \in \mathbb{I}$, and

$$
\limsup _{t \rightarrow \infty} \int_{t_{0}}^{t}\left[\frac{\int_{a}^{b} Q(s, \xi) \mathrm{d} \sigma(\xi)}{2^{\alpha-1}} \delta^{\alpha}(s)-\left(1+\frac{p_{0}{ }^{\alpha}}{\tau_{0}}\right)\left(\frac{\alpha}{\alpha+1}\right)^{\alpha+1} \frac{\eta^{\prime}(s)}{\delta(s) r^{1 / \alpha}[\eta(s)]}\right] \mathrm{d} s=\infty
$$

then (1.1) is oscillatory.

Proof Let $x$ be a nonoscillatory solution of (1.1). Without loss of generality, we assume that there exists a $t_{1} \in \mathbb{I}$ such that $x(t)>0, x[\tau(t)]>0$, and $x[g(t, \xi)]>0$ for all $t \geq t_{1}$ and $\xi \in[a, b]$. Then $z(t)>0$. As in the proof of Theorem 2.1, we get (2.2). By virtue of (1.1), we have (2.3). Thus, $r\left|z^{\prime}\right|^{\alpha-1} z^{\prime}$ is nonincreasing. Now we have two possible cases for the sign of $z^{\prime}$ : (i) $z^{\prime}<0$ eventually, or (ii) $z^{\prime}>0$ eventually.

(i) Suppose that $z^{\prime}(t)>0$ for $t \geq t_{2} \geq t_{1}$. Then, by the proof of Theorem 2.2, we obtain a contradiction to $(2.15)$.

(ii) Suppose that $z^{\prime}(t)<0$ for $t \geq t_{2} \geq t_{1}$. It follows from (2.2), (2.3), and $g(t, \xi) \leq g(t, b)$ that

$$
\begin{gathered}
\left(-r(t)\left(-z^{\prime}(t)\right)^{\alpha}\right)^{\prime}+\frac{p_{0}^{\alpha}}{\tau_{0}}\left(-r[\tau(t)]\left(-z^{\prime}[\tau(t)]\right)^{\alpha}\right)^{\prime} \\
+\frac{1}{2^{\alpha-1}} z^{\alpha}[g(t, b)] \int_{a}^{b} Q(t, \xi) \mathrm{d} \sigma(\xi) \leq 0 .
\end{gathered}
$$

We define the function $u$ by

$$
u(t):=-\frac{r(t)\left(-z^{\prime}(t)\right)^{\alpha}}{z^{\alpha}[\eta(t)]}, \quad t \geq t_{2}
$$

Then $u(t)<0$. Noting that $r\left(-z^{\prime}\right)^{\alpha}$ is nondecreasing, we get

$$
z^{\prime}(s) \leq \frac{r^{1 / \alpha}(t)}{r^{1 / \alpha}(s)} z^{\prime}(t), \quad s \geq t \geq t_{2}
$$

Integrating this inequality from $\eta(t)$ to $l$, we obtain

$$
z(l) \leq z[\eta(t)]+r^{1 / \alpha}(t) z^{\prime}(t) \int_{\eta(t)}^{l} \frac{\mathrm{d} s}{r^{1 / \alpha}(s)}
$$

Letting $l \rightarrow \infty$, we have

$$
0 \leq z[\eta(t)]+r^{1 / \alpha}(t) z^{\prime}(t) \delta(t)
$$


That is,

$$
-\delta(t) \frac{r^{1 / \alpha}(t) z^{\prime}(t)}{z[\eta(t)]} \leq 1
$$

Thus, we get by (2.29)

$$
-\delta^{\alpha}(t) u(t) \leq 1
$$

Similarly, we define another function $v$ by

$$
v(t):=-\frac{r[\tau(t)]\left(-z^{\prime}[\tau(t)]\right)^{\alpha}}{z^{\alpha}[\eta(t)]}, \quad t \geq t_{2}
$$

Then $v(t)<0$. Noting that $r\left(-z^{\prime}\right)^{\alpha}$ is nondecreasing and $\tau(t) \leq t$, we get

$$
r(t)\left(-z^{\prime}(t)\right)^{\alpha} \geq r[\tau(t)]\left(-z^{\prime}[\tau(t)]\right)^{\alpha}
$$

Thus, $0<-v(t) \leq-u(t)$. Hence, by $(2.30)$, we see that

$$
-\delta^{\alpha}(t) v(t) \leq 1
$$

Differentiating (2.29), we obtain

$$
u^{\prime}(t)=\frac{\left(-r(t)\left(-z^{\prime}(t)\right)^{\alpha}\right)^{\prime} z^{\alpha}[\eta(t)]+\alpha r(t)\left(-z^{\prime}(t)\right)^{\alpha} z^{\alpha-1}[\eta(t)] z^{\prime}[\eta(t)] \eta^{\prime}(t)}{z^{2 \alpha}[\eta(t)]} .
$$

By (2.3) and $\eta(t) \geq t$, we have $z^{\prime}[\eta(t)] \leq(r(t) / r[\eta(t)])^{1 / \alpha} z^{\prime}(t)$, and so

$$
u^{\prime}(t) \leq \frac{\left(-r(t)\left(-z^{\prime}(t)\right)^{\alpha}\right)^{\prime}}{z^{\alpha}[\eta(t)]}-\alpha \frac{\eta^{\prime}(t)}{r^{1 / \alpha}[\eta(t)]}(-u(t))^{(\alpha+1) / \alpha}
$$

Similarly, we see that

$$
v^{\prime}(t) \leq \frac{\left(-r[\tau(t)]\left(-z^{\prime}[\tau(t)]\right)^{\alpha}\right)^{\prime}}{z^{\alpha}[\eta(t)]}-\alpha \frac{\eta^{\prime}(t)}{r^{1 / \alpha}[\eta(t)]}(-v(t))^{(\alpha+1) / \alpha} .
$$

Combining (2.33) and (2.34), we get

$$
\begin{aligned}
u^{\prime}(t)+\frac{p_{0}^{\alpha}}{\tau_{0}} v^{\prime}(t) \leq & \frac{\left(-r(t)\left(-z^{\prime}(t)\right)^{\alpha}\right)^{\prime}}{z^{\alpha}[\eta(t)]}+\frac{p_{0}^{\alpha}}{\tau_{0}} \frac{\left(-r[\tau(t)]\left(-z^{\prime}[\tau(t)]\right)^{\alpha}\right)^{\prime}}{z^{\alpha}[\eta(t)]} \\
& -\alpha \frac{\eta^{\prime}(t)}{r^{1 / \alpha}[\eta(t)]}(-u(t))^{(\alpha+1) / \alpha}-\frac{\alpha p_{0}^{\alpha}}{\tau_{0}} \frac{\eta^{\prime}(t)}{r^{1 / \alpha}[\eta(t)]}(-v(t))^{(\alpha+1) / \alpha} .
\end{aligned}
$$

Using (2.28), (2.35), and $g(t, b) \leq \eta(t)$, we obtain

$$
\begin{aligned}
u^{\prime}(t)+\frac{p_{0}^{\alpha}}{\tau_{0}} v^{\prime}(t) \leq & -\frac{\int_{a}^{b} Q(t, \xi) \mathrm{d} \sigma(\xi)}{2^{\alpha-1}}-\alpha \frac{\eta^{\prime}(t)}{r^{1 / \alpha}[\eta(t)]}(-u(t))^{(\alpha+1) / \alpha} \\
& -\frac{\alpha p_{0}{ }^{\alpha}}{\tau_{0}} \frac{\eta^{\prime}(t)}{r^{1 / \alpha}[\eta(t)]}(-v(t))^{(\alpha+1) / \alpha} .
\end{aligned}
$$


Multiplying (2.36) by $\delta^{\alpha}(t)$ and integrating the resulting inequality from $t_{2}$ to $t$, we have

$$
\begin{gathered}
u(t) \delta^{\alpha}(t)-u\left(t_{2}\right) \delta^{\alpha}\left(t_{2}\right)+\alpha \int_{t_{2}}^{t} \frac{\delta^{\alpha-1}(s) \eta^{\prime}(s) u(s)}{r^{1 / \alpha}[\eta(s)]} \mathrm{d} s+\alpha \int_{t_{2}}^{t} \frac{\eta^{\prime}(s) \delta^{\alpha}(s)}{r^{1 / \alpha}[\eta(s)]}(-u(s))^{(\alpha+1) / \alpha} \mathrm{d} s \\
+\frac{p_{0}{ }^{\alpha}}{\tau_{0}} v(t) \delta^{\alpha}(t)-\frac{p_{0}^{\alpha}}{\tau_{0}} v\left(t_{2}\right) \delta^{\alpha}\left(t_{2}\right)+\frac{\alpha p_{0}{ }^{\alpha}}{\tau_{0}} \int_{t_{2}}^{t} \frac{\delta^{\alpha-1}(s) \eta^{\prime}(s) v(s)}{r^{1 / \alpha}[\eta(s)]} \mathrm{d} s \\
+\frac{\alpha p_{0}{ }^{\alpha}}{\tau_{0}} \int_{t_{2}}^{t} \frac{\eta^{\prime}(s) \delta^{\alpha}(s)}{r^{1 / \alpha}[\eta(s)]}(-v(s))^{(\alpha+1) / \alpha} \mathrm{d} s+\int_{t_{2}}^{t} \frac{\int_{a}^{b} Q(s, \xi) \mathrm{d} \sigma(\xi)}{2^{\alpha-1}} \delta^{\alpha}(s) \mathrm{d} s \leq 0 .
\end{gathered}
$$

Set

$$
\begin{aligned}
& A:=-\left[\frac{\eta^{\prime}(t) \delta^{\alpha}(t)}{r^{1 / \alpha}[\eta(t)]}\right]^{(\alpha+1) / \alpha} u(t) \quad \text { and } \\
& B:=\left[\frac{\alpha}{\alpha+1} \frac{\delta^{\alpha-1}(t) \eta^{\prime}(t)}{r^{1 / \alpha}[\eta(t)]}\left[\frac{\eta^{\prime}(t) \delta^{\alpha}(t)}{r^{1 / \alpha}[\eta(t)]}\right]^{-\alpha /(\alpha+1)}\right]^{\alpha} .
\end{aligned}
$$

Using inequality (2.14), we get

$$
\frac{\delta^{\alpha-1}(t) \eta^{\prime}(t) u(t)}{r^{1 / \alpha}[\eta(t)]}+\frac{\eta^{\prime}(t) \delta^{\alpha}(t)}{r^{1 / \alpha}[\eta(t)]}(-u(t))^{(\alpha+1) / \alpha} \geq-\frac{1}{\alpha}\left(\frac{\alpha}{\alpha+1}\right)^{\alpha+1} \frac{\eta^{\prime}(t)}{\delta(t) r^{1 / \alpha}[\eta(t)]} .
$$

Similarly, we set

$$
\begin{aligned}
& A:=-\left[\frac{\eta^{\prime}(t) \delta^{\alpha}(t)}{r^{1 / \alpha}[\eta(t)]}\right]^{(\alpha+1) / \alpha} v(t) \quad \text { and } \\
& B:=\left[\frac{\alpha}{\alpha+1} \frac{\delta^{\alpha-1}(t) \eta^{\prime}(t)}{r^{1 / \alpha}[\eta(t)]}\left[\frac{\eta^{\prime}(t) \delta^{\alpha}(t)}{r^{1 / \alpha}[\eta(t)]}\right]^{-\alpha /(\alpha+1)}\right]^{\alpha} .
\end{aligned}
$$

Then we have by (2.14)

$$
\frac{\delta^{\alpha-1}(t) \eta^{\prime}(t) v(t)}{r^{1 / \alpha}[\eta(t)]}+\frac{\eta^{\prime}(t) \delta^{\alpha}(t)}{r^{1 / \alpha}[\eta(t)]}(-v(t))^{(\alpha+1) / \alpha} \geq-\frac{1}{\alpha}\left(\frac{\alpha}{\alpha+1}\right)^{\alpha+1} \frac{\eta^{\prime}(t)}{\delta(t) r^{1 / \alpha}[\eta(t)]} .
$$

Thus, from (2.30) and (2.32), we find

$$
\begin{aligned}
& \int_{t_{2}}^{t}\left[\frac{\int_{a}^{b} Q(s, \xi) \mathrm{d} \sigma(\xi)}{2^{\alpha-1}} \delta^{\alpha}(s)-\left(1+\frac{p_{0}{ }^{\alpha}}{\tau_{0}}\right)\left(\frac{\alpha}{\alpha+1}\right)^{\alpha+1} \frac{\eta^{\prime}(s)}{\delta(s) r^{1 / \alpha}[\eta(s)]}\right] \mathrm{d} s \\
& \quad \leq u\left(t_{2}\right) \delta^{\alpha}\left(t_{2}\right)+\frac{p_{0}{ }^{\alpha}}{\tau_{0}} v\left(t_{2}\right) \delta^{\alpha}\left(t_{2}\right)+1+\frac{p_{0}{ }^{\alpha}}{\tau_{0}}
\end{aligned}
$$

which contradicts (2.27). This completes the proof.

With a proof similar to the proof of Theorems 2.4 and 2.5, we obtain the following result.

Theorem 2.6 Suppose we have $\left(\mathrm{H}_{1}\right)-\left(\mathrm{H}_{5}\right),(1.2),(1.4)$, and let $\tau(t) \leq t, g(t, a) \geq \tau(t)$ for $t \in \mathbb{I}$, and $g(t, \xi) \leq g(t, b)$ for $\xi \in[a, b]$. Assume also that there exists a real-valued function $\rho \in C^{1}(\mathbb{I},(0, \infty))$ such that $(2.26)$ is satisfied. If there exists a real-valued function $\eta \in C^{1}(\mathbb{I}, \mathbb{R})$ such that $\eta(t) \geq t, \eta(t) \geq g(t, b), \eta^{\prime}(t)>0$ for $t \in \mathbb{I}$, and (2.27) holds, then (1.1) is oscillatory. 


\section{Applications and discussion}

In this section, we provide three examples to illustrate the main results.

Example 3.1 Consider the second-order neutral functional differential equation

$$
[x(t)+x(t-2 \pi)]^{\prime \prime}+\int_{-\frac{5 \pi}{2}}^{\frac{\pi}{2}} x[t+\xi] \mathrm{d} \xi=0, \quad t \geq 10 .
$$

Let $\alpha=1, a=-5 \pi / 2, b=\pi / 2, r(t)=1, p(t)=1, \tau(t)=t-2 \pi, q(t, \xi)=1, g(t, \xi)=t+\xi, \sigma(\xi)=$ $\xi$, and $\rho(t)=1$. Then $Q(t, \xi)=\min \{q(t, \xi), q(\tau(t), \xi)\}=1, g^{\prime}(t, a)=1, g(t, a)=t-5 \pi / 2 \leq t+\xi$ for $\xi \in[-5 \pi / 2, \pi / 2]$, and $g(t, a) \leq \tau(t) \leq t$. Moreover, letting $\tau_{0}=1$, then

$$
\begin{aligned}
& \limsup _{t \rightarrow \infty} \int_{t_{0}}^{t}\left[\frac{\rho(s) \int_{a}^{b} Q(s, \xi) \mathrm{d} \sigma(\xi)}{2^{\alpha-1}}-\frac{1}{(\alpha+1)^{\alpha+1}}\left(1+\frac{p_{0}^{\alpha}}{\tau_{0}}\right) \frac{r[g(s, a)]\left(\rho_{+}^{\prime}(s)\right)^{\alpha+1}}{\left(\rho(s) g^{\prime}(s, a)\right)^{\alpha}}\right] \mathrm{d} s \\
& \quad=3 \pi \limsup _{t \rightarrow \infty} \int_{10}^{t} \mathrm{~d} s=\infty .
\end{aligned}
$$

Hence, by Theorem 2.2, (3.1) is oscillatory. As a matter of fact, one such solution is $x(t)=$ $\sin t$.

Example 3.2 Consider the second-order neutral functional differential equation

$$
[x(t)+t x(t-\beta)]^{\prime \prime}+\int_{0}^{1} \frac{\xi+1}{t} x[t+\xi] \mathrm{d} \xi=0, \quad t \geq 1
$$

where $\beta \geq 0$ is a constant. Let $\alpha=1, a=0, b=1, r(t)=1, p(t)=t, \tau(t)=t-\beta, q(t, \xi)=(\xi+$ $1) / t, g(t, \xi)=t+\xi, \sigma(\xi)=\xi$, and $\rho(t)=1$. Then $Q(t, \xi)=\min \{q(t, \xi), q(\tau(t), \xi)\}=(\xi+1) / t$, $g(t, a)=g(t, 0)=t \leq t+\xi$ for $\xi \in[0,1], \tau(t)=t-\beta \leq t$, and $g(t, a) \geq \tau(t)$ for $t \geq 1$. Further, setting $h(t)=t+1$,

$$
\begin{aligned}
& \phi(t)=\frac{\alpha p^{\prime}[h(t)] h^{\prime}(t)}{p[h(t)]}-\frac{\tau^{\prime \prime}(t)}{\tau^{\prime}(t)}=\frac{1}{t+1}, \\
& \zeta(t)=\frac{\rho_{+}^{\prime}(t)}{\rho(t)}+\phi(t)=\frac{1}{t+1},
\end{aligned}
$$

and

$$
\varphi(t)=\left(\frac{\rho_{+}^{\prime}(t)}{\rho(t)}\right)^{\alpha+1}+\frac{p^{\alpha}[h(t)]\left(\zeta_{+}(t)\right)^{\alpha+1}}{\tau^{\prime}(t)}=\frac{1}{t+1} .
$$

Therefore, we have

$$
\begin{aligned}
& \limsup _{t \rightarrow \infty} \int_{t_{0}}^{t} \rho(s)\left[\frac{\int_{a}^{b} Q(s, \xi) \mathrm{d} \sigma(\xi)}{2^{\alpha-1}}-\frac{r[\tau(s)] \varphi(s)}{(\alpha+1)^{\alpha+1}\left(\tau^{\prime}(s)\right)^{\alpha}}\right] \mathrm{d} s \\
& \quad=\limsup _{t \rightarrow \infty} \int_{1}^{t}\left[\int_{0}^{1} \frac{\xi+1}{s} \mathrm{~d} \xi-\frac{1}{4(s+1)}\right] \mathrm{d} s=\limsup _{t \rightarrow \infty} \int_{1}^{t}\left[\frac{3}{2 s}-\frac{1}{4(s+1)}\right] \mathrm{d} s=\infty .
\end{aligned}
$$

Hence, (3.2) is oscillatory due to Theorem 2.3. 
Example 3.3 Consider the second-order neutral functional differential equation

$$
\left[t^{2}(x(t)+p(t) x(t-\beta))^{\prime}\right]^{\prime}+\int_{0}^{1}(\xi+1) x[t+\xi] \mathrm{d} \xi=0, \quad t \geq 1,
$$

where $0 \leq p(t) \leq p_{0}, p_{0}$ and $\beta$ are positive constants. Let $\alpha=1, a=0, b=1, r(t)=t^{2}$, $\tau(t)=t-\beta, q(t, \xi)=\xi+1, g(t, \xi)=t+\xi, \sigma(\xi)=\xi, \rho(t)=1$, and $\eta(t)=t+1$. Then $Q(t, \xi)=$ $\min \{q(t, \xi), q(\tau(t), \xi)\}=\xi+1, \tau_{0}=1, g(t, a)=g(t, 0)=t \leq t+\xi$ for $\xi \in[0,1], \tau(t)=t-\beta \leq t$, $g(t, a) \geq \tau(t)$ for $t \geq 1$, and $\delta(t)=1 / t$. Further,

$$
\begin{aligned}
& \limsup _{t \rightarrow \infty} \int_{t_{0}}^{t}\left[\frac{\rho(s) \int_{a}^{b} Q(s, \xi) \mathrm{d} \sigma(\xi)}{2^{\alpha-1}}-\frac{1}{(\alpha+1)^{\alpha+1}}\left(1+\frac{p_{0}{ }^{\alpha}}{\tau_{0}}\right) \frac{r[\tau(s)]\left(\rho_{+}^{\prime}(s)\right)^{\alpha+1}}{\left(\tau_{0} \rho(s)\right)^{\alpha}}\right] \mathrm{d} s \\
& \quad=\frac{3}{2} \limsup _{t \rightarrow \infty} \int_{1}^{t} \mathrm{~d} s=\infty
\end{aligned}
$$

and

$$
\begin{aligned}
& \limsup _{t \rightarrow \infty} \int_{t_{0}}^{t}\left[\frac{\int_{a}^{b} Q(s, \xi) \mathrm{d} \sigma(\xi)}{2^{\alpha-1}} \delta^{\alpha}(s)-\left(1+\frac{p_{0}^{\alpha}}{\tau_{0}}\right)\left(\frac{\alpha}{\alpha+1}\right)^{\alpha+1} \frac{\eta^{\prime}(s)}{\delta(s) r^{1 / \alpha}[\eta(s)]}\right] \mathrm{d} s \\
& \quad=\left(\frac{3}{2}-\frac{1+p_{0}}{4}\right) \limsup _{t \rightarrow \infty} \int_{1}^{t} \frac{\mathrm{d} s}{s+1}=\infty, \quad \text { if } p_{0}<5 .
\end{aligned}
$$

Hence, by Theorem 2.6, (3.3) is oscillatory when $0 \leq p(t) \leq p_{0}<5$.

Remark 3.1 In this paper, we establish some new oscillation theorems for (1.1) in the case where $p$ is finite or infinite on $\mathbb{I}$. The criteria obtained extend the results in [22] and improve those reported in [19]. Similar results can be presented under the assumption that $0<\alpha \leq 1$. In this case, using [5, Lemma 2], one has to replace $Q(t, \xi):=$ $\min \{q(t, \xi), q(\tau(t), \xi)\}$ with $Q(t, \xi):=2^{\alpha-1} \min \{q(t, \xi), q(\tau(t), \xi)\}$ and proceed as above. It would be interesting to find another method to investigate (1.1) in the case where $g(\tau(t), \xi) \not \equiv \tau[g(t, \xi)]$.

\section{Competing interests}

The authors declare that they have no competing interests

\section{Authors' contributions}

All authors contributed equally to this work. They all read and approved the final version of the manuscript.

\section{Author details}

${ }^{1}$ Qingdao Technological University, Feixian, Shandong 273400, P.R. China. ${ }^{2}$ Department of Mathematics, Faculty of Electrical Engineering and Informatics, Technical University of Košice, Letná 9, Košice, 042 00, Slovakia.

\section{Acknowledgements}

The authors express their sincere gratitude to the anonymous referees for the careful reading of the original manuscript and useful comments that helped to improve the presentation of the results and accentuate important details.

Received: 16 January 2014 Accepted: 6 March 2014 Published: 24 Mar 2014

\section{References}

1. Hale, JK: Theory of Functional Differential Equations. Springer, New York (1977)

2. Agarwal, RP, Bohner, M, Li, W-T: Nonoscillation and Oscillation Theory for Functional Differential Equations. Dekker, New York (2004)

3. Agarwal, RP, Grace, SR, O'Regan, D: Oscillation Theory for Difference and Functional Differential Equations. Kluwer Academic, Dordrecht (2000) 
4. Baculíková, B, Džurina, J: Oscillation theorems for second order neutral differential equations. Comput. Math. Appl. 61, 94-99 (2011)

5. Baculíková, B, Džurina, J: Oscillation theorems for second order nonlinear neutral differential equations. Comput. Math. Appl. 62, 4472-4478 (2011)

6. Baculíková, B, Lacková, D: Oscillation criteria for second order retarded differential equations. Stud. Univ. Zilina Math. Ser. 20, 11-18 (2006)

7. Baculíková, B, Li, T, Džurina, J: Oscillation theorems for second-order superlinear neutral differential equations. Math. Slovaca 63, 123-134 (2013)

8. Candan, T: The existence of nonoscillatory solutions of higher order nonlinear neutral equations. Appl. Math. Lett. 25, 412-416 (2012)

9. Candan, T, Dahiya, RS: Existence of nonoscillatory solutions of higher order neutral differential equations with distributed deviating arguments. Math. Slovaca 63, 183-190 (2013)

10. Candan, T, Karpuz, B, Öcalan, Ö: Oscillation of neutral differential equations with distributed deviating arguments. Nonlinear Oscil. 15, 65-76 (2012)

11. Dix, JG, Karpuz, B, Rath, R: Necessary and sufficient conditions for the oscillation of differential equations involving distributed arguments. Electron. J. Qual. Theory Differ. Equ. 2011, 1-15 (2011)

12. Džurina, J, Hudáková, D: Oscillation of second order neutral delay differential equations. Math. Bohem. 134, 31-38 (2009)

13. Hasanbulli, M, Rogovchenko, YuV: Oscillation criteria for second order nonlinear neutral differential equations. Appl. Math. Comput. 215, 4392-4399 (2010)

14. Karpuz, B, Öcalan, Ö, Öztürk, S: Comparison theorems on the oscillation and asymptotic behaviour of higher-order neutral differential equations. Glasg. Math. J. 52, 107-114 (2010)

15. Li, T, Agarwal, RP, Bohner, M: Some oscillation results for second-order neutral differential equations. J. Indian Math. Soc. 79, 97-106 (2012)

16. Li, T, Han, Z, Zhang, C, Li, H: Oscillation criteria for second-order superlinear neutral differential equations. Abstr. Appl. Anal. 2011, 367541 (2011)

17. Li, T, Rogovchenko, YuV, Zhang, C: Oscillation of second-order neutral differential equations. Funkc. Ekvacioj 56 , 111-120 (2013)

18. Li, T, Sun, S, Han, Z, Han, B, Sun, Y: Oscillation results for second-order quasi-linear neutral delay differential equations. Hacet. J. Math. Stat. 42, 131-138 (2013)

19. Li, T, Thandapani, E: Oscillation of second-order quasi-linear neutral functional dynamic equations with distributed deviating arguments. J. Nonlinear Sci. Appl. 4, 180-192 (2011)

20. Li, WN: Oscillation of higher order delay differential equations of neutral type. Georgian Math. J. 7. 347-353 (2000)

21. Şenel, MT, Candan, T: Oscillation of second order nonlinear neutral differential equation. J. Comput. Anal. Appl. 14 $1112-1117$ (2012)

22. Sun, $\mathrm{S}, \mathrm{Li}, \mathrm{T}, \mathrm{Han}, \mathrm{Z}, \mathrm{Li}, \mathrm{H}$ : Oscillation theorems for second-order quasilinear neutral functional differential equations. Abstr. Appl. Anal. 2012, 819342 (2012)

23. Thandapani, E, Piramanantham, V: Oscillation criteria of second order neutral delay dynamic equations with distributed deviating arguments. Electron. J. Qual. Theory Differ. Equ. 2010, 1-15 (2010)

24. Wang, P: Oscillation criteria for second order neutral equations with distributed deviating arguments. Comput. Math. Appl. 47, 1935-1946 (2004)

25. Xu, Z, Weng, P: Oscillation of second-order neutral equations with distributed deviating arguments. J. Comput. Appl. Math. 202, 460-477 (2007)

26. Ye, L, Xu, Z: Interval oscillation of second order neutral equations with distributed deviating argument. Adv. Dyn. Syst. Appl. 1, 219-233 (2006)

27. Yu, Y, Fu, X: Oscillation of second order neutral equation with continuous distributed deviating argument. Rad. Mat. 7, 167-176 (1991)

28. Zhang, C, Agarwal, RP, Bohner, M, Li, T: Oscillation of second-order nonlinear neutral dynamic equations with noncanonical operators. Bull. Malays. Math. Sci. Soc. (2014, in press)

29. Zhang, C, Baculíková, B, Džurina, J, Li, T: Oscillation results for second-order mixed neutral differential equations with distributed deviating arguments. Math. Slovaca (2014, in press)

30. Zhao, J, Meng, F: Oscillation criteria for second-order neutral equations with distributed deviating argument. Appl. Math. Comput. 206, 485-493 (2008)

10.1186/1687-2770-2014-68

Cite this article as: $L i$ et al.: Oscillatory behavior of second-order nonlinear neutral differential equations with distributed deviating arguments. Boundary Value Problems 2014, 2014:68 